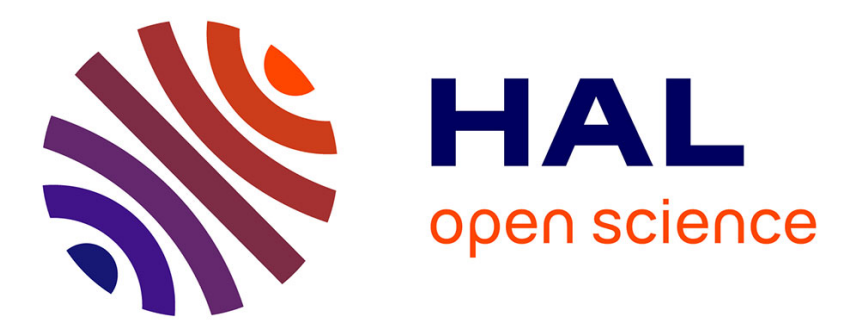

\title{
Motion Estimation and Characterization in Premature Newborns Using Long Duration Video Recordings
}

\author{
Sandie Cabon, Fabienne Porée, Antoine Simon, M Ugolin, O Rosec, G
}

Carrault, P Pladys

\section{- To cite this version:}

Sandie Cabon, Fabienne Porée, Antoine Simon, M Ugolin, O Rosec, et al.. Motion Estimation and Characterization in Premature Newborns Using Long Duration Video Recordings. Innovation and Research in BioMedical engineering, 2017, 38 (4), pp.207-213. 10.1016/j.irbm.2017.05.003 . hal01535540

\author{
HAL Id: hal-01535540 \\ https://hal.science/hal-01535540
}

Submitted on 9 Jun 2017

HAL is a multi-disciplinary open access archive for the deposit and dissemination of scientific research documents, whether they are published or not. The documents may come from teaching and research institutions in France or abroad, or from public or private research centers.
L'archive ouverte pluridisciplinaire HAL, est destinée au dépôt et à la diffusion de documents scientifiques de niveau recherche, publiés ou non, émanant des établissements d'enseignement et de recherche français ou étrangers, des laboratoires publics ou privés. 


\section{Motion estimation and characterization in premature newborns using long duration video recordings}

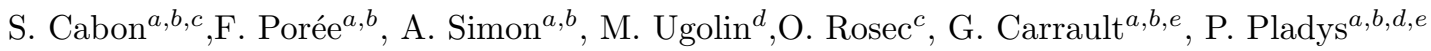

${ }^{a}$ Université de Rennes 1, LTSI, Rennes F-35000, France

${ }^{b}$ INSERM, U1099, Rennes, F-35000, France

${ }^{c}$ Voxygen Health, Rennes, F-35000, France

${ }^{d}$ CHU Rennes, Pôle de pédiatrie médico-chirurgicale et génétique clinique, Rennes F-35000, France

${ }^{e}$ INSERM, CIC-1414, Rennes F-35000, France

Corresponding author:

Fabienne Porée

Université de Rennes 1, LTSI, Rennes F-35000, France

E-mail address: fabienne.poree@univ-rennes1.fr 


\begin{abstract}
Objectives: In the context of neonatal non invasive monitoring, this paper proposes the estimation and characterization of the motion of premature newborns from long duration video recordings.

Material and Methods: A set of 13 videos from 9 different patients, corresponding to 190 hours of recordings, have been studied. An algorithm based on the analysis of changes in the image border has been used to remove intervals artifacted by adults' presence. Then, some features were computed to characterize the baby's motion. The approach was applied to compare two groups of premature newborns, with different severities of prematurity, recorded at the same postmenstrual age.
\end{abstract}

Results: Detection of adults' presence was achieved with $96.8 \%$ of sensitivity. All features were found statistically significant to differentiate the two groups.

Conclusion: This study shows that the automated video monitoring on long periods is achievable and provides relevant information about the premature newborns motion activity.

\title{
1. Introduction
}

A newborn is considered premature when he/she is born before a gestational age (GA) of 37 complete weeks. Causes of prematurity are diverse including multiple pregnancies, infections, hypertensive disease of pregnancy... Worldwide, an estimated 15 million babies are born preterm each year and this number is increasing, ranging from about $5 \%$ in several European countries to $18 \%$ in some African countries [4].

Prematurity is associated with a high risk of mortality, whose primary causes are infection and respiratory failure, and preterm birth outcome is related to the severity of prematurity [19]. Therefore, in the first part of their life, premature newborns are hospitalized in Neonatal Intensive Care Unit (NICU) where their vital functions are monitored such as cardiac, cerebral and respiratory activities.

Additionally to these electrophysiological signals, other types of information have been shown to be relevant to observe newborns' behavior, among them the motion activity. Indeed, studies have demonstrated its ability to detect neurological pathologies. A part of them is based on the General Movement Assessment (GMA) which provides a qualitative motion analysis [1, 3, 8, 12, 15-17]. It has been applied to predict cerebral palsy $[1,15,17]$ or to reflect white matter injury [16]. In the field of neonatal seizures (see [14] for review), motion quantification methods have been applied to represent the motion globally [5] or locally [10, 11]. It has also been shown useful in the context of sleep staging $[9,13]$.

Motor activity information can be extracted from direct sensors like accelerometers [3], electromagnetic sensors [12] or pressure mattress [7], or using video cameras [5, 9-11, 13, 14, 16, 17]. Video 
observation has the advantage to be entirely non invasive and contactless, which limits the risk of infection (one important cause of nosocomial disease in NICU) and preserves the comfort of the newborns. However, it needs to be processed to provide the motion information.

From video recordings, motion analysis was firstly performed manually [9, 16]. However, visual assessment, besides being time consuming is also expert-dependent. More recently, thanks to the improvement in video processing, automated methods $[5,10,11,17]$ have been proposed and combine three main steps: motion extraction, analysis and classification.

This works takes part in the European project Digi-NewB whose objectives are to find new indices to detect sepsis and to evaluate the maturation of the newborn, using different types of observations. Video is one of the considered modalities. Its particularity lies in the fact that very long recordings, up to several days, are acquired in order to detect behavioral modifications. It implies that, for some intervals, the parents or and medical staff are in the field of view. Since these adults interact frequently with the newborn, these intervals have to be automatically detected and removed from the analysis, to ensure that motion series finally contain only the motion of the newborn.

This paper is organized as follows. In section 2, we describe the algorithm implemented in order to automatically detect adults' presence in the videos. Then, the motion series and associated features computation is described. Results are presented in section 3. The interest of the study is shown by comparing two groups of premature newborns, with different severities of prematurity, recorded at the same postmenstrual age (PMA). Discussion and Conclusion are given in section 4.

\section{Material and Methods}

The global framework is given by Figure 1

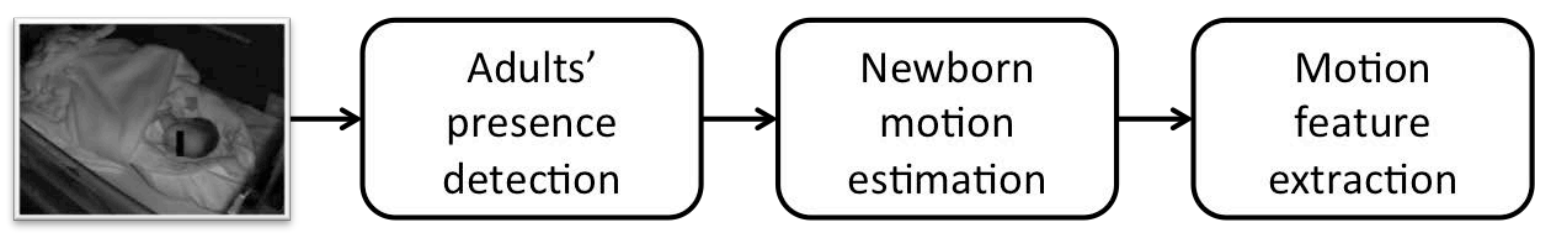

Figure 1: Global framework.

After a description of the data, the different steps involved in the processing are described hereafter.

\subsection{Data acquisition}

Recordings have been performed at the University Hospital Rennes Sud (France). An informed consent was signed by parents for each newborn and validated by the Ethics Committee. An infra-red black and white camera (600x400 pixels, 25 fps) was installed near the bed of the baby in order to observe most of his body. Recordings were mainly performed between $5 \mathrm{pm}$ and $9 \mathrm{am}$. 
The database is composed of 13 videos of 9 premature infants, born between 26 and 36 weeks GA and recorded between 33 and 39 weeks PMA, corresponding to a total of 190 hours of recordings.

\subsection{Medical staff and parents' presence detection method}

Having for objective to monitor the newborns during long periods implies that some intervals will include presence of the parents, rather during the day, but also medical staff, at any time (Figure 2)

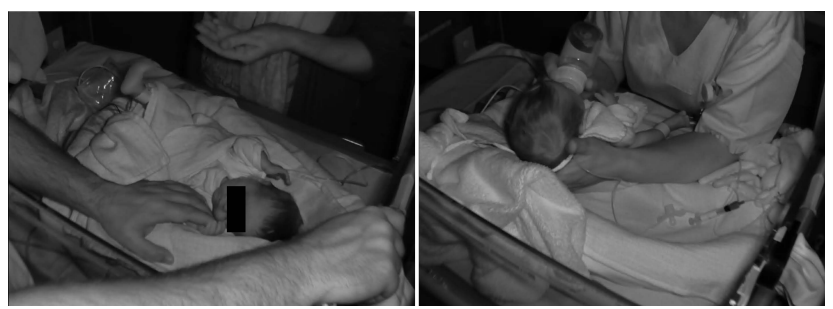

Figure 2: Examples of adults' presence, parents (left) or nurse (right).

To ensure that the motion series contains only motion of the newborn, intervals including adults' presence have to be automatically detected and removed. These intervals can have different durations (from few seconds to tens of minutes)

The main difference between adult's motion and baby motion lies in the fact that, in the first case, it comes from the outside of the scene. This statement led us to develop an algorithm based on the analysis of the change in the image border. Its goal is firstly to detect the adult (medical staff or parent) arrival, and then his/her departure.

\subsubsection{Initialization}

The video analysis is started at the first clean frame, i.e. the first frame without medical staff or parents. The initial reference border is then defined as the border of 20 pixels thickness from each side of this frame (Figure 3). This thickness has been defined according to the area that is not or weakly crossed by the baby.
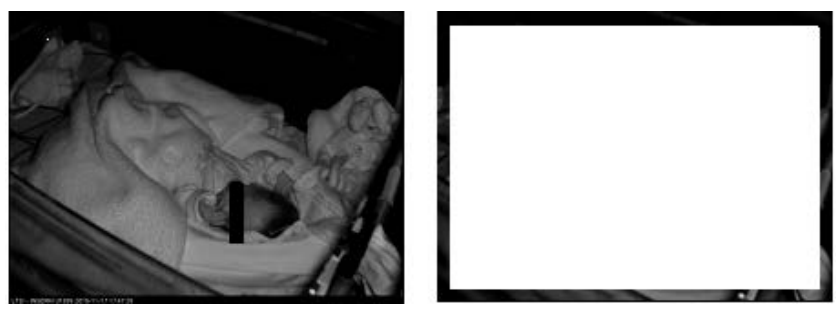

Figure 3: Example of reference border selection. 


\subsubsection{Processing}

Every second, a metric $S(t)$ is computed between the border of the current image $I(t)$ and the reference border Cref:

$$
S(t)=\sum_{p \in C}|I(t, p)-\operatorname{Cref}(p)|
$$

where $C$ is the border area and $I(t, p)$ is the intensity of the pixel $p$ in $I(t)$.

An arrival is detected if $S(t)$ is superior to a threshold $T$ (Figure 4, step 1), which has been set at $6 \%$ of the maximal possible value of the metric, i.e. [number of pixels in $C$ ] x 255 . However, as the adult can have modified the background of the scene (e.g. cable displacement), the amplitude of $S(t)$ can no more be trusted to detect the departure. So, the algorithm waits for the next quiet period, in the border, corresponding to a certainty of departure (step 2). It is defined as a standard deviation of $S(t)$ (over a period of 80 seconds), lower than $0.02 \%$ of the previously defined maximal possible value.

When the quiet period has been detected, the departure time is refined. For this purpose, a temporary border Ctmp is firstly defined on the current image. The metric is then computed backward (step 3), using Ctmp, until the threshold $T$ is reached (step 4). The reference border Cref is then updated and the analysis continues in the forward direction.

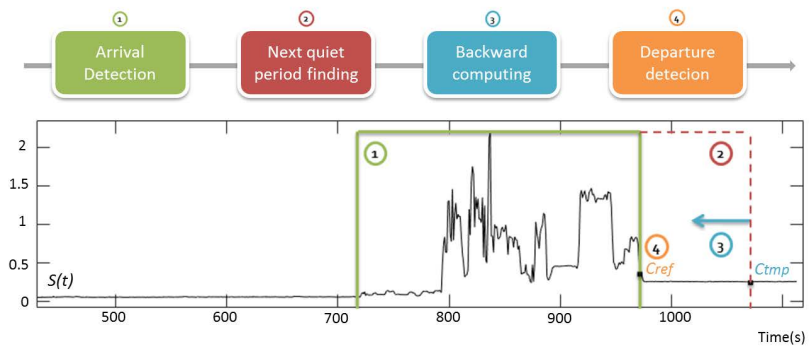

Figure 4: Description of the adults' detection processing.

\subsection{Motion estimation}

There are several approaches for motion estimation in a continuous video stream [18]. Three popular motion information extraction techniques come out: frame differencing, mainly used to identify areas with motion, optical flow and block matching, used to estimate local displacements. In this study, in order to characterize the activity of the newborn, we chose to estimate the global motion information which can be interpreted as total surface in motion. Thus, we used a method based on frame differencing, described below.

Frame differencing. The absolute difference of pixels' intensities between two successive frames $I(t)$ and $I(t-1)$ (Figure 5$)$ 


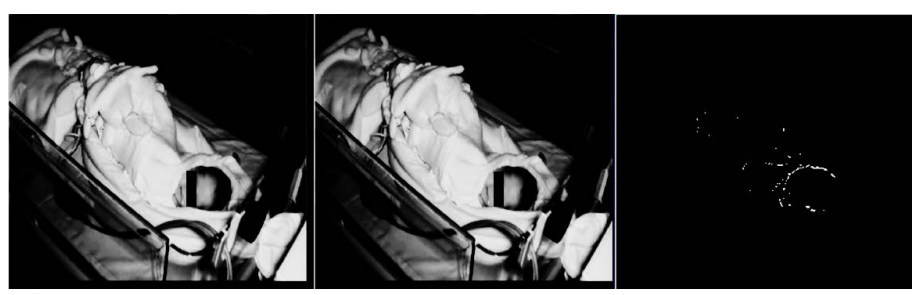

Figure 5: Example of frame differencing.

Morphological opening. A morphological opening with a 3x3 square structuring element was applied to the resulting difference image in order to remove the impulsive noise.

Quantification of the motion. The amount of motion $A(t)$ is obtained by counting the number of pixels with an intensity superior to a threshold $T$ in the resulting image in order to reduce the impact of small intensity variations linked to the camera sensitivity.

The threshold $T$ has been defined by studying the cumulative histogram of video sequences with empty rooms (without baby or adults), resulting to a value of 10 (initial intensity range: $[0,255]$ ).

Median filtering on the whole estimated motion series. The motion series $M(t)$ is finally obtained after applying a median filter (size: 11 samples) on $A(t)$ in order to clear artifacts resulting from the video encoding.

\subsection{Feature extraction}

Two types of features are computed, from the motion series $M(t)$, on 5 -min windows with a $50 \%$ overlap:

- Amplitude features: the mean value $m$, the maximum value max, the standard deviation $s t d$, the median value med and the relative standard deviation $r s d=s t d / m$.

- Activity feature: $p m$, the percentage of time the newborn spent in motion.It is computed by discriminating motion and no motion samples. For this purpose, $M(t)$ is smoothed with a median filter of 51 samples. $\mathrm{pm}$ is computed as the percentage of resulting values greater than 100 (corresponding to about $0.05 \%$ of the size of the frame).

\section{Results}

\subsection{Medical staff and parents' detection}

In order to evaluate the algorithm performances, the adults' periods of presence have been manually annotated on 10 different videos, representing 149 hours of recordings. Then, we compared the manual and automatic detections at each second. For both states "Presence" and "Absence", and considering 
the first one as the positive case, values of sensitivity (Se), specificity (Sp) and global concordance (Conc) have been computed.

The proposed method led to a high specificity of $97.9 \%$ but a lower sensitivity of $90.1 \%$. Errors were due to a late detection of the parent or the medical staff arrival or departure when they appeared only in the back of the frame, corresponding to a very small change in the border. According to our objective, we particularly need to detect all the parents and medical staff presence, i.e. reach a sensitivity as high as possible, without cutting too many analyzable periods. For this purpose, we studied the evolution of the sensitivity and specificity after applying different widening durations to each side of the presence's periods provided by the algorithm (see Figure 6). In order to increase the

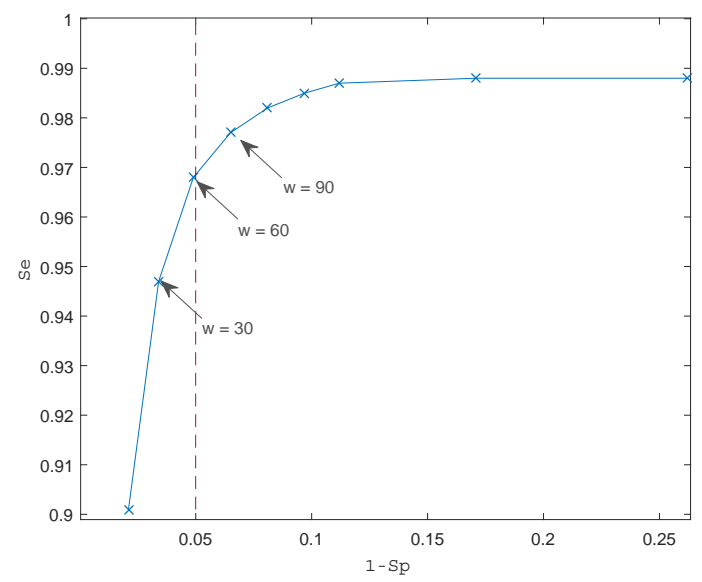

Figure 6: Influence of the widening $w$ on the values of Sp and Se.

algorithm sensitivity without decreasing specificity too much, we chose to apply a widening $w$ of 60 seconds which allows to keep a false positive rate under $5 \%$.

The corresponding performances are presented in Table 1 and show a global concordance of $95.2 \%$, a specificity of $95.1 \%$ and a sensitivity of $96.8 \%$, ranging from $87.7 \%$ to $99.6 \%$. An example of processing is presented by Figure 7 .

\subsection{Newborn activity characterization}

In this study, the aim was to compare two groups of premature newborns, with different severity of prematurity, recorded at a comparable age. As such, we selected a subset of six babies recorded at $37 \pm 2$ weeks PMA:

- three patients born between 26 and 30 weeks (G1);

- three patients born between 32 and 36 weeks (G2). 
Table 1: Performances of the medical staff and parents' detection method with expansion of 60 seconds. Duration in hours. FN: False Negative, TP: True Positive, FP: False Positive and TN: True Negative, in seconds. Se: Sensitivity, Sp: Specificity and Conc: global Concordance, in percent.

\begin{tabular}{ccccccccc}
\hline Video & Duration & FN & TP & FP & TN & Se & Sp & Conc \\
\hline 1 & 17 & 30 & 8463 & 3929 & 47128 & 99.6 & 92.3 & 93.4 \\
2 & 17 & 555 & 10135 & 1068 & 48138 & 94.8 & 97.8 & 97.3 \\
3 & 9 & 3 & 478 & 1354 & 31173 & 99.4 & 95.8 & 95.9 \\
4 & 15 & 265 & 3273 & 2203 & 46674 & 92.5 & 95.5 & 95.3 \\
5 & 14 & 14 & 2686 & 783 & 46717 & 99.5 & 98.4 & 98.4 \\
6 & 16 & 276 & 5316 & 2866 & 49097 & 95.1 & 94.5 & 94.5 \\
7 & 15 & 70 & 3392 & 2648 & 49378 & 98.0 & 94.9 & 95.1 \\
8 & 17 & 97 & 7990 & 2685 & 51976 & 98.8 & 95.1 & 95.6 \\
9 & 13 & 113 & 806 & 4702 & 40949 & 87.7 & 89.7 & 89.7 \\
10 & 16 & 74 & 3338 & 1892 & 52123 & 97.8 & 96.5 & 96.6 \\
\hline Total & 149 & 1497 & 45877 & 24130 & 463353 & 96.8 & 95.1 & 95.2
\end{tabular}

a)

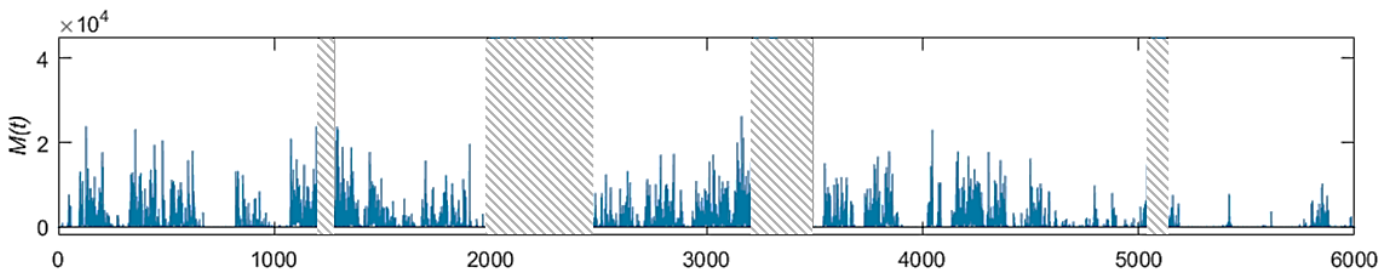

b)

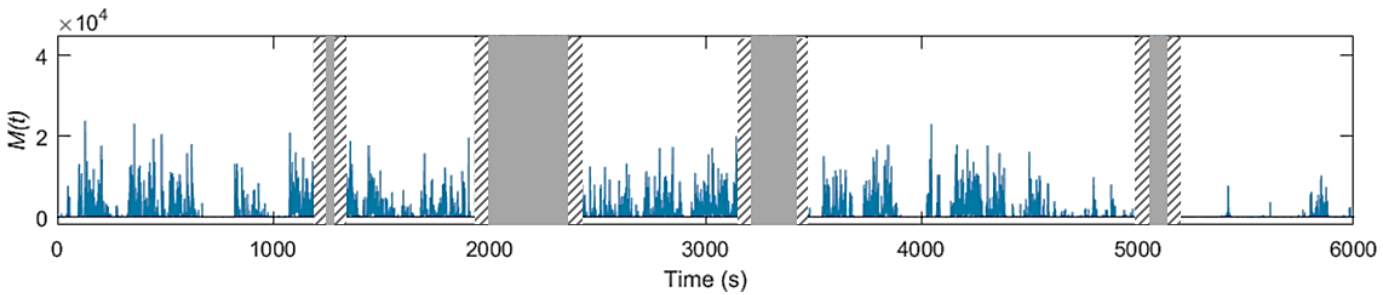

Figure 7: Example of an estimated motion series. a) Manual adult's detection b) Automatic adult's detection before (gray) and after (hatched) the widening. 
The features have then been extracted for each video. The mean video duration was 14 hours, implying, after adult's detection, feature extraction on 1470 windows of 5 minutes (697 for G1, 773 for G2). Figure 8 presents the repartition (mean \pm std) of the feature values for each of the six babies. Then, a Wilcoxon test has been realized on the extracted features in order to compare the two populations. To minimize the repetitive effect, we chose to use the Bootstrap method [20]. Twenty draws of 50 random windows, distributed according to a uniform distribution on the recording duration of each group, have been realized. The p-values have been computed as the median of the p-values of the 20 draws. The results show that all the parameters except the median are statistically significant to differentiate both groups.

In order to check that the results were not due to a side effect linked to differences in recording conditions, we also verified that the same analysis, but performed intra-group, led to no significant parameters. The results for each group demonstrate an homogeneity of the parameters and confirm the previous results.

\section{Discussion and Conclusion}

In this paper, we proposed a process to estimate and characterize the motion of premature newborns from video recordings. In order to handle long duration recordings, we proposed a fully automated method to identify parent's and medical staff periods of presence. The algorithm reached $96.8 \%$ of sensitivity and $95.1 \%$ of specificity.

This study shows that the video monitoring on long periods is achievable and provides relevant information about the patient's behavior. Those preliminary results indicate that the quantity of motion at the same postmenstrual age is greater for the more premature patient. Furthermore, this seems to confirm that the behavioral maturation difference is linked to the time spent in utero [6].

External factors can directly influence some of the proposed amplitude features like the baby size or the recording conditions (shadows, zoom...). However, the relative standard deviation and the percentage of time spent in motion are more robust than the other features because they are not dependent on the amplitude of the motion series. Futhermore, in order to improve the characterization of maturation, robust methods for segmentation will be investigated (e.g. change detection [2]) to extract different activity features such as the number of interval of motion and no motion and statistics on the duration of those intervals (mean, maximum, standard deviation...)

The results could be improved by including an automatic adaptation of the definition of the border area in the adults' detection method. Concerning the characterization of behavior, the results should be strengthened by using a larger dataset. 

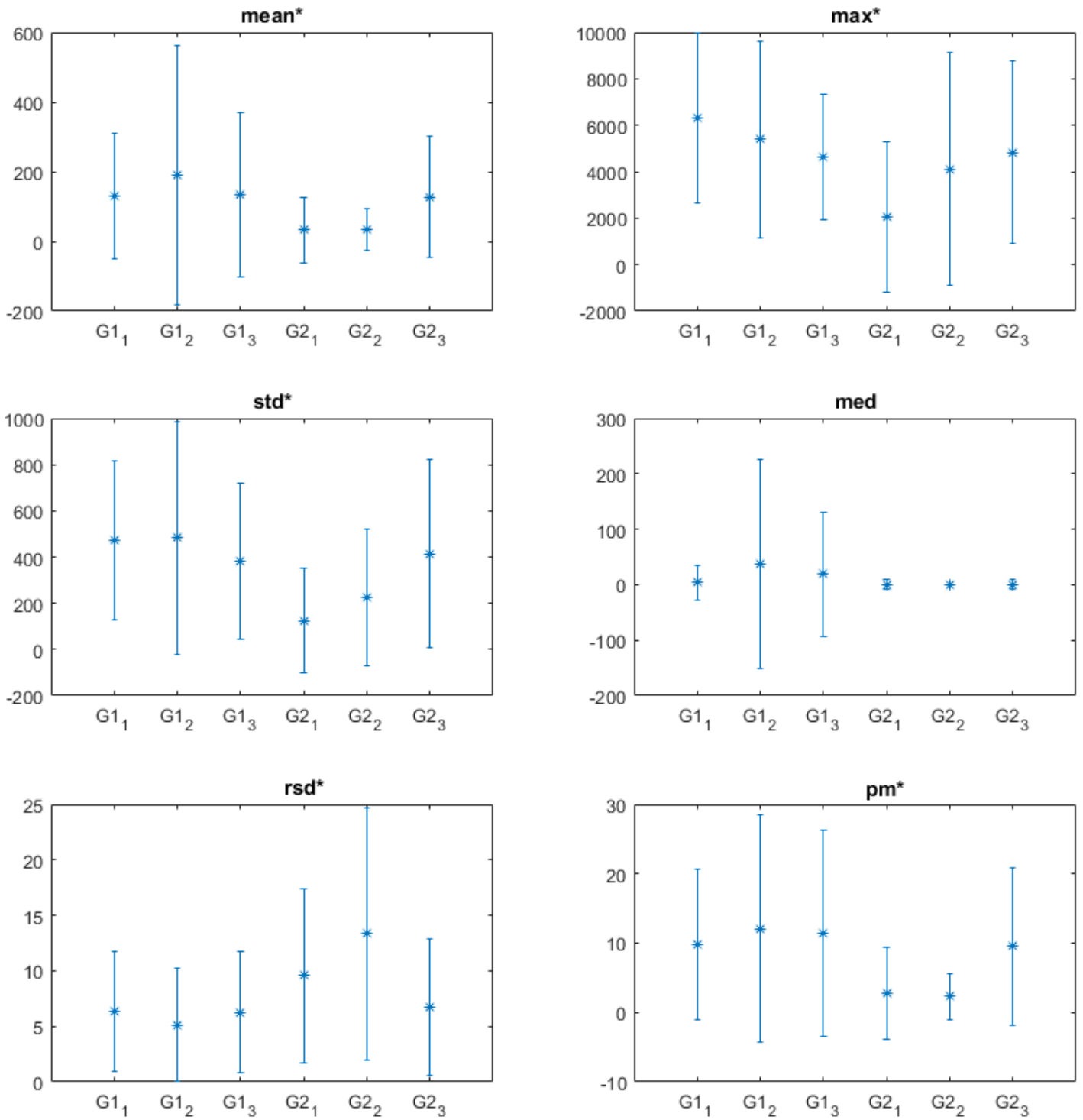

Figure 8: Repartition (mean \pm std) of the values for each baby $\mathrm{Gn}_{\mathrm{i}}$ for each feature, with $\mathrm{n}=$ group number and $\mathrm{i}=$ baby number. The * indicates features with p-value $\leq 0.05$. 


\section{Conflicts of interest}

No conflict of interest.

\section{Acknowledgment}

Results incorporated in this publication were initiated during the VISIM project supported by Région Bretagne, and received funding from the European Union's Horizon 2020 research and innovation program under grant agreement No 689260 (Digi-NewB project).

\section{References}

[1] Adde, L., Rygg, M., Lossius, K., Øberg, G. K., Støen, R., 2007. General movement assessment: predicting cerebral palsy in clinical practise. Early human development 83 (1), 13-18.

[2] Basseville, M., Nikiforov, I. V., et al., 1993. Detection of abrupt changes: theory and application. Vol. 104. Prentice Hall Englewood Cliffs.

[3] Berge, P. R., Adde, L., Espinosa, G., Stavdahl, 2008. ENIGMA - Enhanced interactive general movement assessment. Expert Systems with Applications 34 (4), 2664-2672.

[4] Blencowe, H., Cousens, S., Oestergaard, M. Z., Chou, D., Moller, A.-B., Narwal, R., Adler, A., Garcia, C. V., Rohde, S., Say, L., et al., 2012. National, regional, and worldwide estimates of preterm birth rates in the year 2010 with time trends since 1990 for selected countries: a systematic analysis and implications. The Lancet 379 (9832), 2162-2172.

[5] Cuppens, K., Lagae, L., Ceulemans, B., Huffel, S. V., Vanrumste, B., sep 2010. Automatic video detection of body movement during sleep based on optical flow in pediatric patients with epilepsy. Medical \& Biological Engineering \& Computing 48 (9), 923-931.

[6] Curzi-Dascalova, L., 1992. Physiological correlates of sleep development in premature and fullterm neonates. Neurophysiologie Clinique/Clinical Neurophysiology 22 (2), 151-166.

[7] Donati, M., Cecchi, F., Bonaccorso, F., Branciforte, M., Dario, P., Vitiello, N., 2013. A modular sensorized mat for monitoring infant posture. Sensors (Basel, Switzerland) 14 (1), 510-531.

[8] Einspieler, C., Prechtl, H. F. R., Ferrari, F., Cioni, G., Bos, A. F., nov 1997. The qualitative assessment of general movements in preterm, term and young infants - Review of the methodology. Early Human Development 50 (1), 47-60. 
[9] Fuller, P. W., Wenner, W. H., Blackburn, S., 1978. Comparison between time-lapse video recordings of behavior and polygraphic state determinations in premature infants. Psychophysiology $15(6), 594-598$.

[10] Karayiannis, N. B., Tao, G., Frost, J. D., Wise, M. S., Hrachovy, R. A., Mizrahi, E. M., jul 2006. Automated detection of videotaped neonatal seizures based on motion segmentation methods. Clinical Neurophysiology 117 (7), 1585-1594.

[11] Karayiannis, N. B., Xiong, Y., Frost, J. D., Wise, M. S., Mizrahi, E. M., 2005. Quantifying motion in video recordings of neonatal seizures by robust motion trackers based on block motion models. IEEE Transactions on Biomedical Engineering 52 (6), 1065-1077.

[12] Karch, D., Kim, K. S., Wochner, K., Pietz, J., Dickhaus, H., Philippi, H., sep 2008. Quantification of the segmental kinematics of spontaneous infant movements. Journal of Biomechanics 41 (13), $2860-2867$.

[13] Okada, S., Ohno, Y., Kato-Nishimura, K., Mohri, I., Taniike, M., et al., 2008. Examination of non-restrictive and non-invasive sleep evaluation technique for children using difference images. In: Engineering in Medicine and Biology Society, 2008. EMBS 2008. 30th Annual International Conference of the IEEE. IEEE, pp. 3483-3487.

[14] Pediaditis, M., Tsiknakis, M., Leitgeb, N., dec 2012. Vision-based motion detection, analysis and recognition of epileptic seizures-A systematic review. Computer Methods and Programs in Biomedicine 108 (3), 1133-1148.

[15] Prechtl, H. F., Einspieler, C., Cioni, G., Bos, A. F., Ferrari, F., Sontheimer, D., 1997. An early marker for neurological deficits after perinatal brain lesions. The Lancet 349 (9062), 1361-1363.

[16] Spittle, A. J., Brown, N. C., Doyle, L. W., Boyd, R. N., Hunt, R. W., Bear, M., Inder, T. E., may 2008. Quality of general movements is related to white matter pathology in very preterm infants. Pediatrics 121 (5), e1184-9.

[17] Stahl, A., Schellewald, C., Stavdahl, Ø., Aamo, O. M., Adde, L., Kirkerod, H., jul 2012. An optical flow-based method to predict infantile cerebral palsy. IEEE Transactions on Neural Systems and Rehabilitation Engineering 20 (4), 605-614.

[18] Tekalp, A. M., 2015. Digital video processing. Prentice Hall Press.

[19] Tucker, J., McGuire, W., 2004. Epidemiology of preterm birth. BMJ: British Medical Journal 329 (7467), 675 . 
[20] Zoubir, A. M., Boashash, B., 1998. The bootstrap and its application in signal processing. IEEE signal processing magazine 15 (1), 56-76. 